\title{
El Currículum Simétrico en la Formación de Profesionales de Salud y Alimentación con los Pueblos Originarios: La Experiencia con la Etnia Wixárika de México
}

\author{
The Symmetrical Curriculum in the Training of Health and \\ Food Professionals with the Original Peoples: The Experience \\ with the Wixárika of Mexico
}

\author{
René Cristóbal Crocker * \\ Teresa de Jesús Pérez Patiño \\ José Luis Vázquez Castellanos \\ Patricia Muñoz López \\ Universidad de Guadalajara
}

\begin{abstract}
En la investigación se analiza el proceso de construcción intercultural de la Licenciatura en Salud y Alimentación Intercultural con el pueblo Wixárika de México como parte del proyecto de Universidad Interétnico Cultural que se desarrolla a través de la metodología de investigación acción participativa con los líderes de esta etnia mexicana, con base en la técnica etnográfica de diálogos interétnico culturales. Se construye un perfil de egreso con el enfoque de competencias sustentado en el concepto de Currículum Simétrico y en la Teoría de la Actividad Humana, en donde se recuperan saberes en salud y alimentación tradicionales que se integran de forma crítica con los saberes occidentales. La estructura y el mapa curricular se organizan por módulos, a partir de problemas del mundo real Wixárika y ejes integradores de prácticas profesionales y reflexión científica de la práctica en la medicina y en el sistema productivo de alimentos tradicionales y en el sistema de salud y proyectos productivos occidentales. Se concluye, que la teoría y metodología utilizada es una veta que puede permitir la formación de recursos humanos interculturales con base en el diálogo de saberes en situación de igualdad que permite desarrollar la medicina intercultural en el país.
\end{abstract}

Descriptores: Grupo étnico, México, Salud, Nutrición.

In this work, it's analyzed the curriculum design of bachelor's degree in health science and human feeding from an intercultural view with Wixarika community in Mexico, as part of the project for the construction and creation of the Interethnic Cultural University Wixárika which is guided by the methodology of participative research action with leaders of the Wixarika ethnic, based on cultural interethnic dialogues' ethnographic research technique. The graduate profile is created and based on a competence approach sustained on Activity theory and "Symmetrical Curriculum" concept, of which traditional feeding and health knowledge are recovered to be critically integrated to the occidental knowledge. The curriculum map and structure are organized by the real problems of the Wixarika's world, the integral axes of professional practices and scientific reflection of medicine, traditional food productive systems, the health system and the productive occidental projects. It's concluded that the theory and methodology are a vein that can allow the formation of intercultural human resources based on knowledge dialogue on equal situations that can develop intercultural medicine in the country.

Keywords: Ethnic groups, Mexico, Health, Nutrition.

*Contacto: recricrosa_7@hotmail.com

ISSN: 0718-7378

www.rinace.net/rlei/
Recibido: $\quad 16 / 06 / 2017$

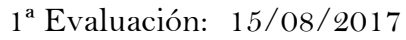

Aceptado: 25/09/2017 


\section{Introducción}

En la investigación se analiza el proceso de construcción del diseño curricular de la Licenciatura en Salud y Alimentación Intercultural, que forma parte de las propuestas generadas por los líderes del pueblo Wixárika para desarrollar la Universidad Intercultural de la Región Wixárika a partir del año 2010, con el propósito de formar profesionales que desarrollen alternativas de solución a los problemas de salud y nutrición presentes en esta población, a través de recuperar los saberes "del costumbre" Wixárika y saberes occidentales, con énfasis en la comunicación intercultural, en el contexto de los rituales y tradiciones propias de la etnia relacionados con el cuidado a la Madre Naturaleza.

La Universidad Intercultural de la Región Wixárika es un proyecto construido por el Pueblo Wixárika con asesoría y apoyo de un grupo de investigadores y educadores de la Universidad de Guadalajara, a partir de la demanda de sus comunidades de desarrollar una propuesta de educación superior vinculada a su cultura y a sus necesidades de desarrollo social, que además contribuya a disminuir la migración de los jóvenes de la etnia, de sus lugares de origen a las grandes ciudades, por falta de opciones educativas y de empleo.

El interés de publicar el presente artículo tiene el propósito de aportar elementos teóricos y metodológicos relacionados con el concepto de currículum simétrico interétnico cultural como un elemento para elaborar propuestas de educación intercultural sustentadas en el diálogo de saberes entre sabios de las comunidades y agentes externos a través de metodologías participativas, ubicando el foco de investigación en la etnia Wixárika, por ser este pueblo el que mantiene altos niveles de empoderamiento entre los pueblos de la Región Occidental de México.

Desde hace 40 años, el Estado Mexicano, ha realizado esfuerzos institucionales para resolver la problemática de salud de los pueblos indígenas de la Región, sin embargo, la situación de salud-enfermedad y la problemática nutricional sigue siendo precaria. El estudio se justifica porque los sistemas de atención a la salud y la alimentación tradicionales y occidentales están divorciados en las comunidades del pueblo Wixárika, debido a que no existe un diálogo de saberes en salud entre ambos, siendo uno de los factores, entre otros, que impactan en la situación precaria de salud de los pueblos originarios. Uno de los factores es la ausencia de recursos humanos de las comunidades que intermedien entre ambos sistemas, por lo que la construcción del diseño curricular en Salud y Alimentación Intercultural, pretende llenar éste vacío educativo y de atención a la salud.

La investigación se fundamenta en el Paradigma Posmoderno de la Complejidad Crítica, que permite integrar las concepciones críticas, en sus visiones modernas y postmodernas. Ello significa, romper con las epistemologías clásicas Positivistas e Interpretativas, que permitan dar cabida a la comprensión de las prácticas de identidad social y de conocimiento de los pueblos originarios y su intencionalidad, de participar con visión emancipadora, en la transformación de su propia realidad y de las políticas educativas universitarias y del Estado (Buenfil, 1995; De Suoza Santos, 2005). Desde esta perspectiva se concibe el currículum como una propuesta político-educativa hegemónica que permea el plan de estudios y las prácticas que realizan los miembros de una institución o comunidad educativa en coherencia o contradicción con el contexto social económico y 
político donde se ubican. En coherencia con esa definición, se ubica al concepto de currículum simétrico interétnico intercultural como un aporte a la educación inclusiva.

La lógica del poder en la complejidad de la educación interétnica cultural en las sociedades capitalistas postcoloniales, en su fase de globalización neoliberal, no es posible analizarla, únicamente desde la lógica de la hegemonía y la reproducción social, ya que, las sociedades actuales han desarrollado múltiples prácticas sociales y poderes políticos. El concepto de poder, presente en la Teoría de la Contrahegemonía, propuesta por Boaventura De Sousa Santos (De Souza Santos, 2006) amplía los límites y el alcance de la noción de poder productivo, mercantil e ideológico, propuesto por los teóricos de la hegemonía y la reproducción social, incorporando otros niveles de análisis, como el espacio doméstico, comunitario y ciudadano en un contexto de mundialización, por lo que estos elementos, se incorporan en el estudio de las prácticas educativas que realiza la etnia Wixárika y los actores universitarios.

Para abordar el objeto de estudio se analizan tres unidades de análisis: a) los elementos culturales, epidemiológicos y pedagógico didácticos que permiten formar recursos humanos en salud del pueblo Wixárika para fundamentar una propuesta curricular intercultural en salud y alimentación; b) las características que debe tener el perfil profesional de egreso intercultural en salud y alimentación y por último, c) las estrategias curriculares que permitan formar a los jóvenes wixaritari con saberes para desarrollar un programa de salud y alimentación intercultural.

Para comprender el problema de estudio, relacionado con la ausencia de un programa interétnico cultural de formación de profesionales de la etnia Wixárika, que establezcan un diálogo de saberes entre el Sistema de Salud Tradicional y el Sistema de Salud Occidental, que coexisten en la región Wixárika y que permita resolver las principales enfermedades de malnutrición y enfermedades crónico-degenerativas de sus comunidades, se conceptualiza a la educación interétnica cultural, como un constructo teórico de los autores que tiene utilidad para comprender los diferentes paradigmas de la educación intercultural, que se implementan en los espacios educativos en donde coexisten las etnias originarias de América con población mestiza, como: las políticas, saberes, prácticas y acciones intencionadas que realizan los sujetos de una institución educativa con fines de aprendizaje interétnico cultural para favorecer la identidad étnica cultural y el desarrollo social de los pueblos originarios, como parte de los procesos formativos para los estudiantes indígenas y no indígenas en situación de igualdad, que permitan el desarrollo de sociedades y estados nación multiétnicos, interculturales y plurilingües (Crocker, 2013).

El concepto es útil, ya que existen diferentes abordajes relacionados con el problema de estudio, que van desde la Educación Multicultural, la Educación Intercultural Funcionalista y la Educación Intercultural Crítica (Dietz, 2007; Williamson, 2004) analiza los paradigmas hegemónicos educativos con grupos excluidos en Estados Unidos de América y Europa (Paradigmas de la Multiculturalidad y la Interculturalidad Funcionalista), señala que en el debate, sobre todo anglosajón, se plantea la necesidad de "multiculturalizar" los sistemas educativos mediante mecanismos de "acción afirmativa" y "discriminación positiva” que permitan "empoderar" (empower) a determinadas minorías étnicas, autóctonas tanto como alóctonas, en sus procesos de identificación, etnogénesis y "emancipación”. En el espacio continental europeo, por el contrario, se percibe la urgencia de desarrollar una educación intercultural, que surge como un medio para integrar y 
asimilar a los inmigrantes, provenientes de mundos culturales diversos, en donde se ignora las necesidades identitarias de las minorías, lo que muestra la incapacidad manifiesta de las sociedades mayoritarias de hacer frente a los nuevos desafíos de la heterogeneidad de los educandos, de la creciente complejidad sociocultural y en resumidas cuentas, de la diversidad como característica de las futuras sociedades europeas.

El Paradigma de la Interculturalidad Crítica, señala Walsh (2007) cuestiona el modelo de sociedad vigente, ya que la interculturalidad funcional asume la diversidad cultural como eje central, apuntalando su reconocimiento e inclusión dentro de la sociedad y el Estado nacional (uninacional por práctica y concepción) y dejando por fuera los dispositivos y patrones de poder institucional estructural -las que mantienen la desigualdad- la Interculturalidad Crítica parte del problema del poder, su patrón de racialización y la diferencia (colonial no simplemente cultural) que ha sido construida en función de ello. El Multiculturalismo Postcolonial y el Interculturalismo Funcional, responden a y parte de los intereses y necesidades de las instituciones sociales; la interculturalidad crítica, en cambio, es una construcción de y desde la gente que ha sufrido un histórico sometimiento y subalternización.

El diseño del Plan de Estudios de la Licenciatura en Salud y Alimentación Humana Intercultural, se desarrolla con base en el concepto de Currículum Simétrico Interétnico Cultural. Sustentado en el Paradigma de la Interculturalidad Crítica (Crocker, 2015) Esta propuesta de construcción curricular se sustenta en establecer una comunicación y diálogo respetuoso entre los saberes y conocimientos de los pueblos indígenas y el conocimiento occidental relacionado con el campo de la salud y la alimentación, que se establece en las Comunidades Emergentes de Conocimiento Intercultural Local (CECIL) en donde participan sabios de la comunidad e investigadores del campo de la salud y la alimentación, que supere la visión de colonialismo del conocimiento que prevalece en las disciplinas de este campo científico.

Hasta ahora, los criterios de validez del conocimiento, las teorías para interpretar la realidad y la transmisión del conocimiento en los diferentes niveles educativos universitarios en el campo de la salud y la alimentación, se sustentan en los parámetros de la modernidad europeo-norteamericana. Como parte de los paradigmas de la Modernidad Científica, se han creado disciplinas como la Etnobotánica y la Antropología Médica, etc. en donde los pueblos indígenas han sido transformados en objetos de estudio, descripción y análisis, que excluyen la posibilidad de auto-comprensión y el planteamiento de soluciones a los problemas de salud y alimentación, por los propios pueblos originarios.

Como parte del Colonialismo y Postcolonialismo Cultural, desde el Siglo XVI se han legitimado los saberes dominantes de los colonizadores y la exclusión de los saberes de los colonizados. La consolidación hegemónica del pensamiento Moderno y Postmoderno a partir del impulso de los intereses del Complejo Médico Industrial y Semillero Norteamericano en América Latina en los últimos sesenta años, ha profundizado el colonialismo cultural y ha contribuido a subalternizar el pensamiento indígena en salud y alimentación, ya que no cumple con los criterios de validez científica, definidos desde los parámetros del conocimiento construidos en Europa y Norteamérica.

Para la innovación curricular de la educación en salud y alimentación Wixárika universitaria es importante preguntarse ¿Cuál es el papel del conocimiento de las etnias de la Región, en la construcción de una propuesta curricular simétrica interétnico cultural alternativa a las políticas hegemónicas del Complejo Médico Industrial Norteamericano y 
Semillero, que hagan viable un sistema de salud y alimentación interétnico, intercultural y plurilingüe que supere el pensamiento hegemónico educativo en salud y alimentación postcolonial?

En la educación universitaria actual para los pueblos indígenas, las propuestas curriculares en salud para formarlos están construidas desde la visión occidental, sin tomar en cuenta la cosmovisión y los saberes en salud ancestrales, lo que está provocando que los egresados de la etnia, al reinsertarse en sus comunidades carezcan de elementos para la comprensión de la problemática en salud en sus pueblos, así como, no tengan una actitud favorable para trabajar de manera colaborativa con los sabios en salud de la etnia Wixárika.

Un elemento central en la construcción del currículum simétrico interétnico cultural para la Licenciatura en Salud y Alimentación Humana Intercultural es la diversificación epistémica, que debe pasar por una fase de colaboración interétnico cultural en la producción de conocimientos, entre diversos y heterogéneos enfoques y actores. De ahí la importancia de redefinir y distinguir conceptual y políticamente el discurso interétnico cultural en términos de diversidad, diferencia y desigualdad, como eje para recuperar los saberes de la etnia Wixárika y combinarlos críticamente con los saberes occidentales que tiendan a la descolonización del currículum y permitan construir el currículum interétnico cultural.

Un elemento innovador de la investigación curricular es la aplicación de un enfoque de Educación Basada en Competencias sustentado en la Teoría de la Actividad Humana (Cole, 1993) Desde esta Teoría aplicada al análisis y construcción de propuestas de transformación educativa en relaciones interétnico culturales participativas, es fundamental la comprensión de la forma cómo se distribuyen los saberes y las cogniciones mentales, así cómo se construyen las prácticas educativas en sociedades inequitativas en contextos postcoloniales, como la que viven los pueblos originarios a partir de la Globalización Neoliberal.

Los pueblos, en contextos de transición sociopolítica priorizan las acciones educativas sustentadas en la actividad práctica para resolver problemas concretos, con base en la construcción de estrategias de identidad sociocultural, en donde el papel de las construcciones mentales sustentadas en la identidad social es fundamental (Kostiuk, 1986).

Los teóricos de la Actividad Humana que se citan en párrafos previos, proponen un abordaje de los objetos de estudio socio-pedagógicos, basada en la idea de una cognición distribuida entre los individuos y un conocimiento construido socialmente, a través, de esfuerzos cooperativos dirigidos a alcanzar objetivos comunes y desarrollados en los entornos culturales, donde la información es objeto de un procesamiento que tiene lugar entre los individuos y las herramientas y artefactos que proporciona la cultura.

La cognición, de acuerdo a estos autores está distribuida en personas, cultura, etnias, mundo social y en el tiempo (lo histórico). Ello significa, que para generar propuestas de educación interétnica culturales es necesario resignificar la historia, la cultura étnica, los saberes y el mundo social.

Para construir propuestas curriculares innovadoras que incorporen los saberes de los pueblos originarios en los espacios universitarios interétnico culturales, es fundamental su recuperación a través de un proceso conjunto de reconstrucción interétnica cultural del conocimiento y sus prácticas sociales (Mato, 2005). 
Con base en los elementos mencionados, para emprender la construcción del currículum simétrico interétnico cultural en salud y alimentación sustentado en enfoque de competencias basadas en la actividad humana cotidiana es imprescindible comenzar por reubicar, reconstruir y relacionar saberes subalternizados y silenciados históricamente, como el saber de los mara'akate (curanderos tradicionales de la etnia) y de los productores de coamil, (sistema tradicional agroecológico de alimentos en donde se combina la siembra de maíz, frijol y calabaza) que permitan interrelacionar los saberes tradicionales que circulan en la sociedad Wixárika y buscar críticamente, su vinculación con los saberes occidentales.

Es necesario generar cauces innovadores para diversificar el conocimiento occidental y monológico que caracteriza a los currícula occidentales hegemónicos en los procesos formativos en medicina y nutrición de las universidades occidentales, para relacionarlos con conocimientos locales, ciencias y saberes alternativos de la etnia Wixárika y otros pueblos indígenas y mestizos de la región, que en su confluencia pueden y deben fertilizarse mutuamente construyendo nuevos cánones diversificados de conocimiento.

La descolonización del conocimiento y los saberes educativos en los espacios universitarios, es un proceso gradual e integrador que tiene que estar sustentado en investigación disciplinar y educativa que sistematice el conocimiento y los saberes educativos del pueblo Wixárika, incorporando la cosmovisión de recreación del conocimiento y los saberes educativos de la etnia, pero integrándolos con la visión occidental de hacer ciencia, realizada principalmente por los intelectuales y sabios de este pueblo y por investigadores occidentales ligados a los intereses políticos de la etnia, a través del desarrollo de Comunidades del Conocimiento Interétnico Cultural Locales en salud y alimentación, como la experiencia de la escuela Kiekari +kitsikapa. (Lugar donde se Aprende con la Madre Naturaleza en idioma Wixarika). Esta ruptura epistemológica, sociológica e ideológica, está relacionada con el papel autocrítico que algunos intelectuales universitarios realizan en sus prácticas científicas, al vincularlas a los intereses de los pueblos indígenas, que han estado excluidos de potencializar su propio conocimiento a partir de los procesos coloniales.

En esta línea de pensamiento, el conocimiento Wixárika en salud y alimentación, aporta elementos de inimaginable valor ecológico y de resistencia cultural al campo del currículum universitario en Ciencias de la Salud, relacionadas con sus sistemas productivos alimentarios, en donde combinan la siembra sustentable del maíz, el frijol y la calabaza con semillas criollas, así como, la ruptura con la visión antropocéntrica de vivir del mundo occidental, en la medida en que se recuperen como contacto cultural interétnico y que se conjunten con otros esfuerzos para el logro de un mejor reparto de la riqueza mundial en dirección de la equidad.

\section{Método}

Para construir la propuesta curricular de la Licenciatura en Salud y Alimentación Intercultural en la Región Wixárika, se aplica un diseño de investigación acción participativa sustentada en la etnografía participante.

Para crear la propuesta del diseño curricular de la Licenciatura, los líderes de la comunidad que coordinan la Universidad Intercultural de la Región Wixárika, convocan a los sabios de la etnia y a los que tienen un cargo dentro de la comunidad: marakames (sabios en salud) 
promotores de salud y nutrición de la etnia, los agricultores que cuentan con huertos y encargados del cuidado de las plantas medicinales; además, los asesores del Programa Intercultural de Agroecología, Salud y Alimentación Wixárika (PIASAW) y estudiantes de las Licenciaturas en Medicina y Nutrición de la Universidad de Guadalajara. En total participan en el proceso de investigación, tres marakames, cinco promotores en salud y nutrición de la etnia, doce agricultores, cuatro investigadores que actúan como asesores externos y ocho estudiantes de la Universidad de Guadalajara.

En la primera fase del proceso, que dura un año, se conforman Comunidades Emergentes de Conocimiento Intercultural Local (CECIL) en donde participan investigadores mestizos y sabios de la comunidad local del campo de la agricultura, la salud y la alimentación, quienes establecen un proceso de diálogo de saberes sobre conocimientos, prácticas y cosmovisión en estos tres campos de discusión, que constituyen uno de los principales problemas que demandaron las comunidades que debería ser resuelto por la Universidad Intercultural de la Región Wixárika

Se conforman 3 CECIL: la primera, para estudiar "el costumbre" Wixárika de producir y consumir alimentos; la segunda, para estudiar la percepción del cuerpo y el espíritu sano y enfermo y la tercera, para estudiar las formas de organizar el sistema de medicina tradicional y los remedios naturales que se utilizan en la terapéutica local.

En la segunda fase, los líderes de la comunidad y los investigadores, realizan un proceso educativo para capacitar a los representantes de la comunidad (Sabios en salud, productores agrícolas y promotores en salud y nutrición intercultural) y a los estudiantes sobre el proceso de diálogo para construir el diseño curricular. Las bases de la propuesta para el diseño curricular fueron construidas en un taller de diálogo interétnico cultural realizado en Kiekari+kitsikapa (Lugar donde se aprende lo que enseña la Madre Naturaleza) en Pueblo Nuevo, Tuapurie, Mezquitic, Jalisco. Durante el taller, se discutió la problemática educativa actual de la etnia Wixárika, se analizó las necesidades y demandas en salud y alimentación intercultural del pueblo Wixárika, la valoración de la factibilidad para realizar la Licenciatura en Tuapurie, se diseñó el perfil de ingreso y egreso hubo participación activa entre los compañeros Wixáritari, en su propio idioma y el intercambio con los asesores mestizos fue realizado por una de las promotoras PIASAW.

En la tercera etapa En la siguiente sesión se construyó la estructura y el mapa curricular de la Licenciatura en Salud y Nutrición Humana Intercultural, el cual fue presentado a los líderes de la Universidad Intercultural de la Región Wixárika para su aprobación y comunicación a los miembros de la comunidad interesados.

\section{Resultados}

Para dar respuesta a las necesidades y demandas de salud y alimentación del Pueblo Wixárika, la comunidad y los líderes locales deciden formar un Licenciado de la Salud Pública Interétnico Cultural como parte del proyecto de construcción de la Universidad Intercultural en la Región Wixárika. Con la formación de este profesional de la salud y la alimentación, se busca articular los esfuerzos de este pueblo y el Estado, para resolver problemas ancestrales de salud-enfermedad y la alimentación, que actualmente están desarticulados o constituyen una imposición simbólica postcolonial del Estado, que anula el diálogo de saberes. 
Desde una perspectiva metodológica, se construye un diseño curricular de tipo mixto, en donde se combina el diseño modular con ejes integradores, a través de un ejercicio de investigación acción participativa. Los principales resultados relacionados de la construcción del perfil profesional y la estructura curricular, se presentan a continuación:

Para fundamentar el perfil profesional de egreso se analizan: la concepción del cuerpo humano y sus relaciones con la naturaleza, las necesidades y demandas asociadas al perfil epidemiológico sociocultural, las prácticas de producción de alimentos y las formas interculturales de resolver los problemas de salud en el sistema de salud tradicional y en el sistema de salud occidental, que están divorciadas y que dificultan el diálogo de saberes entre ambos sistemas de salud y alimentación y que constituyen la situación problematizadora que se trata de resolver con la presente propuesta de diseño curricular, tal como se ejemplifica en los datos que se describen a continuación:

Con respecto a la cosmovisión anatómica y fisiológica del cuerpo humano del pueblo Wixárika, se concluye: los habitantes de la etnia tienen una concepción biocultural del organismo que recupera la energía, a través de una vinculación de los sistemas o espíritus corporales con la Madre Naturaleza a través del Kupuri o Espíritu Mayor, intermediados a través de rituales de acercamiento con sus lugares simbólicos ubicados en los cuatro puntos cardinales: el Padre Sol (Tayau Tau) ubicado en Wirikuta en los desiertos de San Luís Potosí en el Oeste; la Diosa del Mar (Haramara) ubicada en San Blas, Nayarit en el Océano Pacífico, en el Este; la Diosa de los Mares del Sur (Xapamiyameta) ubicada en la Isla de los Alacranes en el Lago de Chapala, cerca de Guadalajara; con el Dios de las Aguas del Norte (Utakame), ubicado en los bosques del Norte en el Estado de Durango, los cuales se comunican con el centro del universo Wixárika, donde habita el Dios del Fuego (Tatewari). La comunicación ritual con los dioses de los lugares sagrados está mediada por plantas alucinógenas como el Peyote (Cactácea recolectada en los desiertos del Norte de México) la caza del venado y rituales al Maíz, como planta sagrada.

La relación de los lugares sagrados con el cuerpo humano la establecen a través de cinco espíritus o sistemas anatomo-fisiológicos corporales: El Espíritu para Sentir (ligado a los sentidos del gusto, piel y audición en la visión occidental) el Espíritu de Ver (ligado a la visión periférica y central en la visión occidental) el Espíritu de Comer (ligado al aparato digestivo y sus anexos, el aparato cardiovascular y genitourinario en la cosmovisión occidental) el Espíritu de Moverse (ligado al aparato musculo-esquelético y sistema nervioso periférico) y el Espíritu de Pensar (vinculado al Sistema Nervioso Central). La semiótica y el abordaje de intercultural de los cuadros sindromáticos del perfil de salud enfermedad y de morir de la etnia, tienen relación con esta visión de analizar al ser humano y sus vínculos con el entorno natural, diferente a la visión antropocéntrica del ser humano, hegemónica en las escuelas de ciencias de la salud del mundo occidental.

Los wixáritari (gentilicio del pueblo Wixárika) distinguen dos tipos de enfermedades: las originarias de la sierra y las traídas por los españoles y sus descendientes mestizos. Las primeras, son aquellas que forman parte de su cosmovisión y que requieren de la medicina tradicional para su curación; en cambio, las segundas, deben ser tratadas con la medicina occidental y tradicional, en donde los problemas se aborden a través de un diálogo de saberes entre la concepción de salud-enfermedad de los sabios en salud indígenas, sin considerarlo pensamiento mágico y las visiones más avanzadas de la salud pública occidental, que son respetuosas de la cultura tradicional y sus recursos terapéuticos. 
El perfil epidemiológico de salud-enfermedad y el proceso alimentario nutricio actual del pueblo Wixárika y las formas de respuesta médico social del sistema de salud tradicional y occidental, desde una perspectiva histórica y sociocultural se caracterizan por los siguientes problemas:

1) Enfermedades "del costumbre" Wixárika, que están vinculadas al no cumplimiento de las tradiciones, que provoca el castigo de los dioses, por ejemplo: El Maxariet+, que está relacionada con infecciones y desnutrición en niños y adultos, asociada con no cumplir el ritual de ir de cacería del venado; el Kierixiya, estados psicóticos relacionados con no realizar el ritual a la planta-Dios del Kieri. Todas estas enfermedades necesitan el apoyo del Mara'akame (sacerdote-curandero) para su tratamiento.

2) Enfermedades de los "Teiwari" o de los mestizos, que llegaron con la vinculación de los pueblos indígenas de la región con el modelo de desarrollo occidental. Ejemplos: obesidad, diabetes, hipertensión arterial, etc. que están relacionadas con las enfermedades crónicodegenerativas, asociadas al consumo de azúcar simples en comunidades con presencia del gen ahorrador, relacionado con períodos de hambre crónica, como las que padece el pueblo Wixárika y que para su prevención, diagnóstico y tratamiento necesitan combinarse los esfuerzos del sistema de salud occidental con el sistema de salud tradicional.

3) Problemas asociados a la soberanía para la seguridad y nutricional alimentaria: se identifica que la producción de alimentos se realiza con el Sistema de Coamil, en donde se asocia la producción ecológica de maíz, frijol y calabaza y en los últimos diez años se incorporan las huertas familiares agroecológicas interculturales. En lo relacionado con la disponibilidad alimentaria, se identifican problemas de escases de agua para regar la siembra y disminución de la calidad del suelo, situaciones en las cuales es necesaria la aplicación de tecnologías agroecológicas apropiadas. El consumo se realiza con alimentos provenientes de la producción familiar y algunas veces de la compra externa de alimentos. Existen procesos de aculturación en el consumo alimentario, por lo que es necesaria la orientación acerca de productos saludables de acuerdo al poder adquisitivo y para que el comprador realice la selección de alimentos que le aporten beneficios a su salud y que el dinero no se gaste en compra de alimentos que contribuyan al desarrollo de enfermedades crónico-degenerativas, como los alimentos refinados e industrializados.

4) Con relación a las demandas del pueblo Wixárika en atención a la salud y la alimentación, se identifica que la atención en los servicios de salud occidentales no son la primera elección de los wixáritari para diagnosticar y tratar las enfermedades, su sistema de salud se basa en la curación por medio de plantas medicinales y consultas con los mara'akame para la curación de las "enfermedades del costumbre". El sistema de salud occidental no comparte los principios tradicionales de diagnosticar y tratar, por lo cual la atención a los wixáritari no se brinda de manera apropiada, aunado a esto, existe problema en la comunicación y la referencia de casos problema de mestizos como de wixáritari.

La medicina tradicional no cuenta con los mismos recursos con los que cuenta la medicina occidental para su desarrollo y las prácticas tradicionales no son valoradas de acuerdo al impacto que produce su práctica en la población. Esto pone en situación de menosprecio a las prácticas de salud de las etnias, por lo cual es importante comprender y poner en práctica el enfoque intercultural y que exista la participación activa de la medicina tradicional. 
Con base en la problemática identificada y los fundamentos pedagógicos planteados en el marco de referencia, se construye el siguiente perfil de competencias de egreso para el Licenciado en Salud y Alimentación Humana Intercultural: 1) Es un el profesional que actúa con ética y valores profesionales de la medicina y la nutrición conservando y desarrollando su identidad étnico-cultural; 2) Previene enfermedades durante el ciclo de la vida a partir de la promoción y protección de la salud 3) Diagnostica y trata holísticamente las "enfermedades del costumbre" y las Teiwari por medio de la comunicación intercultural con el equipo de salud y personajes de la medicina tradicional, a través de comprender los síndromes y terapéuticas de ambos sistemas de salud utilizando el idioma propio y el español; 4) Organiza sistemas de referencia de casos problema entre la medicina tradicional y el sistema de salud occidental, con bases interculturales críticas; 5) Organiza y produce alimentos, al igual que medicinas con las plantas medicinales de su comunidad y promueve la autosuficiencia con respeto a la madre naturaleza; 6) Elabora programas de salud y nutrición con una visión intercultural; 7) Desarrolla su proyecto de trabajo en el campo de la salud y nutrición mediante planeación estratégica a través de la organización, razonamiento, priorización, reflexión crítica y con capacidad de trabajar en equipo; 8) Estudia las prácticas en salud y nutrición del sistema de salud tradicional de sus comunidades para conservarlas y mejorarlas aplicando diversas metodologías científicas de abordaje de la realidad, incluidas las que utilizan los sabios de su comunidad para analizar los problemas de salud; 9) Se autoforma y actualiza de manera permanente en el campo de la salud y la alimentación intercultural con actitudes de inclinación por la lectura y el estudio permanente.

Con base en el perfil de competencias de egreso se elabora una estructura curricular modular con un eje integrador de prácticas profesionales (figura 1) Los módulos se organizan en tres grupos de saberes: los relacionados con el aprendizaje de competencias éticas, culturales y de comunicación social intercultural en salud y alimentación; los módulos que aportan saberes para el aprendizaje de competencias interculturales de anatomía, fisiología-semiólogía y terapéutica para el abordaje integral del individuo sano y enfermo, así como la organización de un sistema de salud intercultural; por último, los módulos relacionados con la promoción, gestión y desarrollo de proyectos y de sistemas de salud y alimentación interculturales en comunidades.

El eje integrador articula las prácticas profesionales y la investigación y se organiza en cinco fases: En la primera, el alumno articula los saberes teórico-metodológicos básicos de las profesiones de la salud con prácticas vivenciales y laboratoriales; en la segunda fase, el estudiante se forma en saberes básicos de la profesión en salud y alimentación, realizando prácticas e investigación en espacios sociales comunitarios, en el sistema de salud y alimentación tradicional y en el sistema de salud y alimentación occidental de las instituciones de la región.

En la tercera fase, el alumno aprende las competencias para la gestión de servicios interculturales en salud y alimentación; en la cuarta fase, el alumno desarrolla y emprende su propio proyecto, articulado con las necesidades de salud y alimentación de sus comunidades, la cosmovisión de curarse y alimentarse de acuerdo a su tradición y las necesidades de desarrollo del sistema de salud y alimentación intercultural; por último, el alumno realiza un servicio social comunitario, en donde implementa su proyecto productivo. 


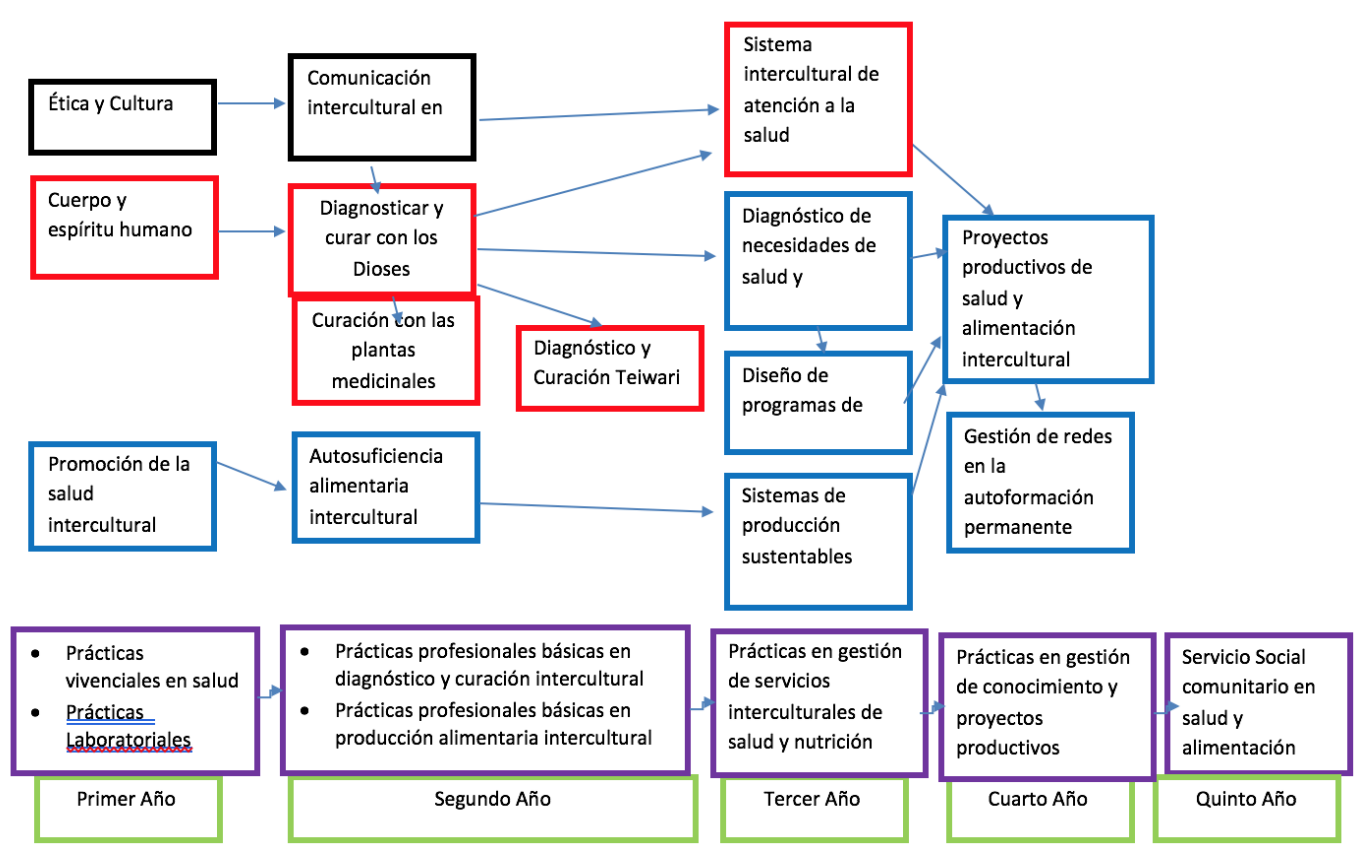

Figura 1. Estructura y mapa curricular por módulos y ejes integradores

Fuente: Elaboración propia.

\section{Discusión}

La construcción curricular de la Licenciatura en Salud y Alimentación Humana Interétnico Cultural que se analiza, se fundamenta en lo pedagógico en la propuesta innovadora de Currículum Simétrico Interétnico Cultural, en donde no existen antecedentes publicados al respecto, por lo que se considera un modelo original de diseño curricular intercultural, por lo que constituye el principal aporte de la investigación.

En la innovación curricular ha sido importante la integración dialéctica e histórica de la cultura, como un elemento de identidad étnica y las necesidades sociales, que contribuyan al desarrollo integral de la etnia Wixárika en dirección de la descolonización del conocimiento y la equidad, como ejes para construir el diseño curricular que se analiza, necesidad surgida de la demanda de los líderes de la etnia que coordinan el Proyecto de Universidad Interétnica Cultural, quienes propusieron, la creación de la Licenciatura en Salud y Alimentación Intercultural y la participación de los sabios tradicionales en salud y alimentación en sus comunidades en la construcción del diseño curricular.

Un elemento central de la propuesta educativa interétnico cultural, es la construcción curricular en donde los saberes occidentales y de los pueblos originarios, son valorados en igualdad, en dirección de descolonizar el currículum que permea la educación universitaria actual.

Para lograr el currículum simétrico interétnico cultural en salud y alimentación intercultural, fue necesario sistematizar inicialmente los saberes y el conocimiento de los pueblos originarios y contrastarlos con los saberes occidentales, a través, de criterios de validación epistémica múltiples, en donde, exista un diálogo entre los sabios indígenas, los profesionales indígenas y mestizos, así como, investigadores y asesores educativos con experiencia curricular intercultural, acción realizada a través de las Comunidades Emergentes de Conocimiento Intercultural Local actividad realizada al inicio del proceso 
de investigación en las comunidades de la Sierra Wixárika, en el contexto de la Universidad Interétnica Cultural.

En un segundo momento, se contrastan los saberes construidos a través del diálogo interétnico cultural, con el desarrollo de los campos disciplinares producto del desarrollo científico tecnológico, para buscar propuestas que adecuadas al desarrollo de las fuerzas productivas locales, que permitan potenciar el desarrollo económico endógeno, en donde los alumnos generen sus propias propuestas productivas y de empleo local en la región, que evite que los jóvenes abandonen sus comunidades y los espacios naturales vinculados a su sistema productivo y rituales, lo que atenta con la existencia misma del pueblo Wixárika

Estos saberes integrados y validados con criterios epistemológicos múltiples, vinculados a las necesidades y demandas de desarrollo económico-social, a la cosmovisión de ser humano de las comunidades indígenas y al desarrollo de la ciencia y tecnología local y regional, son la base para construir perfiles profesionales con pertinencia local, regional y global.

En la construcción del perfil de egreso, fundamentado en las necesidades y la cultura en salud y alimentación Wixárika que permea la estructura curricular del plan de estudios que se analiza, se propicia una ruptura con los enfoques tradicionales, que sustentan los diseños curriculares positivistas de disciplinas aisladas y se elabora una propuesta modular con ejes integradores, en donde, la actividad humana como práctica social y profesional, la reflexión crítico-científica de la práctica y la identidad étnica y cultural, son los elementos centrales.

En los enfoques de educación por competencias que se implementan en las instituciones universitarias convencionales, sustentados en paradigmas conductistas y constructivistas, predominan las competencias transversales y profesionales, surgidas de la visión occidental y de los mercados laborales para integrar a los egresados a los procesos globales y a las profesiones, construidas a partir de las necesidades de reproducción de las fuerzas productivas para fortalecer el mercado de las profesiones.

En la presente propuesta curricular, se desarrollan competencias socioculturales para que los estudiantes asuman un posicionamiento crítico con respecto a su cosmovisión y su identidad social, que les permita desarrollar la cultura en salud y alimentación propia, a la vez de incorporar elementos de otras culturas, a través de aprender a dialogar en situación de igualdad entre los saberes propios y los saberes occidentales, a través de integrarse simultáneamente a experiencias en el sistema de salud y alimentación de su vida cotidiana y a los sistemas de salud y alimentación de las instituciones de salud y alimentación occidentales. En esa dirección, son importantes elementos innovadores relacionados con el aprendizaje en la práctica reflexiva, fundamentado en los aportes teóricos de la Teoría de la Actividad Humana y el concepto de Eje Integrador, que articula la práctica profesional con los módulos teórico-metodológicos a lo largo del proceso formativo del estudiante.

Con base en la experiencia de planeación curricular en el campo de la salud y la alimentación humana intercultural y al análisis de las dificultades para su implementación, las estrategias educativas para la formación de profesionales en los espacios educativos interétnico-culturales, que se proponen a partir de la investigación deberán estar sustentadas en: 
a) La actividad práctica profesional aplicada al análisis y solución de problemas reales del desarrollo social y cultural de los pueblos, principalmente de los pueblos originarios de la región mesoamericana, sus relaciones con el mundo occidental y la reflexión críticocientífica con las teorías y saberes propios y el conocimiento occidental.

b) Desarrollar la competencia para la creación y gestión de proyectos productivos, que sean pertinentes con las necesidades y problemas del desarrollo social y de las fuerzas productivas locales, que les permitan propiciar fuentes de empleo productivo en sus comunidades, desde la educación universitaria.

c) Debido a las dificultades de comunicación presencial, porque los pueblos originarios viven en sus zonas de resistencia de difícil acceso y no cuentan con una planta académica para iniciar los proyectos educativos interétnico-culturales, un elemento central en el proceso formativo es la educación semi-presencial y a distancia, a través, de medios cibernéticos, por lo que deberán desarrollar los medios tecnológicos, la infraestructura y los recursos medios propios, para establecer una estrategia educativa para el aprendizaje en medios virtuales interétnico culturales, aspecto que deberá desarrollarse en otras investigaciones para la educación inclusiva.

d) En la selección y formación del personal académico para la formación de profesionales en la Región Wixárika y otras propuestas propias de los pueblos mesoamericanos, debe prevalecer la prioridad de incorporar profesionales de los pueblos indígenas, así como, incorporar a los sabios indígenas para el desarrollo de saberes de la etnia para promover la cultura Wixárika en los espacios educativos, un elemento propuesto por los sabios indígenas que participaron en la elaboración de la propuesta

A pesar de que el estudio y la propuesta, aportan elementos que pueden ser generalizables a las instituciones educativas en salud y alimentación y a los pueblos originarios de la región latinoamericana, cada país, pueblo e institución universitaria, tiene sus propias estrategias identitarias y políticas para la educación interétnico cultural, que deben ser tomadas en cuenta para la construcción de diseños curriculares para los pueblos originarios.

En la investigación es importante destacar el papel de sujeto social de las comunidades del pueblo Wixárika, quienes constituyen un actor social de primer orden, en la discusión del futuro de la educación superior que se debe implementar en las universidades públicas en América Latina, que permitan que las instituciones educativas participen de manera protagónica en la discusión del papel de la universidad en la descolonización del conocimiento y en el desarrollo de los pueblos de la región. Este elemento es una discusión que se generó con los participantes indígenas y mestizos en la experiencia que se presenta, ya que no sólo es importante interculturalizar la educación para los pueblos originarios, sino que también, es necesario incorporar los aportes de los pueblos originarios al currículum occidental.

Desde los aportes epistemológicos recuperados y la reconstrucción crítica realizada por los participantes en el estudio, la perspectiva de la Complejidad Crítica utilizada en la investigación, es un paradigma que permite el análisis y solución de problemas complejos, como el de la educación con los pueblos indígenas, lo que ha permitido generar un espacio de discusión del pensamiento y cosmovisión con los pueblos originarios, para valorar sus aportes al conocimiento educativo e innovar la educación universitaria en dirección de la equidad y el respeto a la naturaleza, en momentos, en que la crisis social y ambiental 
planetaria, son problemas fundamentales en la discusión, que permita a los estados nacionales y a la sociedad civil, construir modelos para mantener la vida de los ecosistemas en el futuro, sin exclusiones de países, clases sociales, etnias y género.

\section{Agradecimientos}

Los autores agradecen a las comunidades de la etnia Wixárika la posibilidad de recrear el pensamiento educativo para desarrollar el currículo intercultural en el campo de la salud y la alimentación.

\section{Referencias}

Buenfil, R. (1995). Horizonte postmoderno y configuración social. México: CESU-UNAM.

Cole, M. (1993). Enfoque histórico-cultural de la cognición distribuida. Buenos Aires: Amorrortu editores.

Crocker, R. (2013). El proyecto de creación de la universidad interétnica cultural en la región Wixárika. Diálogos sobre Educación, 11, 1-18.

Crocker, R. (2015). Educación universitaria con los pueblos originarios. Guadalajara: Ediciones de la Noche.

De Suoza Santos, B. (2005). La universidad en el siglo XXI: Para una reforma democrática y emancipadora de la universidad. Ciudad de México: CIICH-UNAM.

De Souza Santos, B. (2006). Renovar la teoría crítica y la emancipación social. Buenos Aires: CLACSO.

Dietz, G. (2007). La interculturalidad. Entre el "empoderamiento" de minorías y la "gestión" de la diversidad. puntos de vista. Cuadernos del Observatorio de las Migraciones y de la Convivencia Intercultural de la Ciudad de Madrid, 3, 27-44.

Kostiuk, G. S. (1986). Algunos aspectos de la relación recíproca entre educación y desarrollo de la personalidad. Madrid: Editorial Akal.

Mato, D. (2005). Interculturalidad, producción de conocimientos y prácticas socioeducativas. Caracas: Alceu.

Walsh, C. (2007). Interculturalidad Crítica y pedagogía de-colonial. En C. Walsh, Educación intercultural hoy en América Latina (pp. 5-10). Quito: Universidad Andina Simón Bolivar.

Williamson, G. (2004). ¿Educación multicultural, educación intercultural bilingüe, educación indígena o educación intercultural? Cuadernos Interculturales, 3, 23-24.

\section{Breve CV de los autores}

\section{René Cristóbal Crocker Sagastume}

Médico con especialidad en Pediatría, Profesor Normalista en Educación Bilingüe MayaEspañol, Maestría en Investigación en Ciencias de la Educación y Doctorado en Investigación Educativa Aplicada. Profesor investigador titular, Instituto Regional de Investigación en Salud Pública, Centro Universitario de Ciencias de la Salud, Universidad de Guadalajara. Director de Investigación y Posgrado de Ciencias de la Salud, Universidad Guadalajara Lamar. Línea de investigación: Cultura y Educación en Salud, 
Alimentación y Ambiente. ORCID ID: OOOO-0001-9425-2126. Email: recricrosa_7@hotmail.com

\section{Teresa de Jesús Pérez Patiño}

Cirujana Dentista, Maestría en Ciencias de la Salud Ambiental, Candidata a Doctora en Ciencias de la Salud Ocupacional. Universidad de Guadalajara Profesor Asociado, Departamento de Salud Pública. Universidad de Guadalajara Coordinadora de Docencia en Pregrado, Departamento de Salud Pública. Universidad de Guadalajara Profesor de la Cátedra de Salud Ambiental y Salud Pública, Escuela de Medicina del Centro Universitario de Ciencias de la Salud, Universidad de Guadalajara. Línea de investigación: Cultura y Educación en Salud, Alimentación y Ambiente. ORCID ID: 0000-0002-36400650.Email: patite@hotmail.com

\section{José Luis Vázquez Castellanos}

Médico Cirujano, Especialidad en Epidemiología Aplicada, Maestría en Medicina Social, Doctor en Ciencias Socio-Médicas. Profesor Investigador Titular, Departamento de Salud Pública, Centro Universitario de Ciencias de la Salud, Universidad de Guadalajara. Profesor de Epidemiología, Escuela de Medicina del Centro Universitario de Ciencias de la Salud, Universidad de Guadalajara. Profesor del Doctorado en Ciencias de la Salud Ambiental, Universidad de Guadalajara Departamento de Epidemiología Hospital General Regional 110, Instituto Mexicano del Seguro Social. Guadalajara, Jalisco, México Línea de investigación: Epidemiología y Educación en Salud, Alimentación y Ambiente. ORCID ID: 0000-0002-7874-9746. Email: luisvazmx@yahoo.com.mx

\section{Patricia Muñoz López}

Licenciada en Nutrición. Maestrante de Ciencias Sociales, con orientación en Estudios Sociopolíticos, Centro Universitario de Ciencias Sociales y Humanidades de la Universidad de Guadalajara. Profesora de la Licenciatura en Nutrición de la Universidad de Guadalajara. Línea de investigación: Políticas para la Soberanía y seguridad alimentaria. ORCID ID: 0000-0001-9127-571X. Email: patriciamunozlo@hotmail.com 